UDK UDK 630*228(234.422 Bjelašnica)(253)

\title{
STAND STRUCTURE, DEAD WOOD AND FLORISTIC COMPOSITION OF A PRISTINE FOREST STAND OF BEECH AND FIR COMPARED TO A MANAGED FOREST IN THE DINARIC MOUNTAIN BJELAŠNICA
}

\author{
Komparativna analiza strukture, mrtvog drveta i florističkog sastava prašume i \\ gospodarske šume bukve i jele na planini Bjelašnici \\ Ćemal Višnjić ${ }^{1}$, Sead Vojniković ${ }^{1}$, Besim Balić ${ }^{1}$, Faruk Mekić ${ }^{1}$, Sead Ivojević ${ }^{1}$
}

\begin{abstract}
This paper presents the results of a comparative analysis of the structural and vegetation characteristics of "Ravna vala" pristine (virgin) forest and the managed forest of beech and fir (with spruce). Pristine forest "Ravna vala" is located in the central part of Bosnia and Herzegovina, about $20 \mathrm{~km}$ southwest of Sarajevo on Bjelašnica Mountain, at an altitude from 1280 to $1450 \mathrm{~m}$. The managed forest of beech and fir with spruce, which was used for comparison, borders "Ravna vala" and has the same habitat conditions: climate, orography and land, and the similar structural characteristics and composition of tree species as the pristine forest.

Beech and fir communities (Abieti-Fagetum illyricum Treg. 1957) dominate but spruce can also be found in the area of research. Noble hardwoods such as sycamore and elm are found both individually or in small groups in depressions in deeper soils.

Both the pristine forest and the managed forest show the selective diameter structure. However, the great differences between the pristine forest and the managed forest are final diameter classes. The thickest tree in the pristine forest has the breast diameter of $145 \mathrm{~cm}$, while the diameter of the thickest tree in the industrial forest is $70 \mathrm{~cm}$. The volume of the stands is $770 \mathrm{~m}^{3} / \mathrm{ha}$ in the pristine forest and it is two times larger than in the managed forest. The volume of dead wood in the pristine forest is $170 \mathrm{~m}^{3} / \mathrm{ha}$ and it is five times larger than in the managed forest. While stumps and thicker branches are the most common in the stock of dead trees in the managed forest, the most common in the stock of dead wood in the pristine forest are tall stumps as well as lying and standing dead trees. Floristic researches have shown that selective management does not reflect negatively on floristic diversity, but ground vegetation is richer in species in the managed forest. Shannon's diversity index and evenness index of vascular flora have higher values in the managed forest. Unlike floristic diversity, the stand diversity index of JÄHNE \& DOHRENBUSCH (1997) shows higher values in the pristine forest than in the managed forest.
\end{abstract}

Key words: pristine forest, diversity, dead wood, floristic composition

\footnotetext{
${ }^{1}$ Faculty of Forestry University of Sarajevo
} 


\section{INTRODUCTION - Uvod}

There has been more research about sustainable forest management in recent years. In this regard, the management systems are optimized in order to preserve and enhance the structure, floristic composition, and biodiversity, and thereby the stability, longevity and naturalness of forest ecosystems. It can be achieved with a management system which does not include radical silviculture, but those which imitate the nature. This manner of management is known as "Close-to-nature-forestry" (Schütz, 1986) in the scientific circles and practice. It involves some fundamental postulates: natural regeneration, selection and cutting of individual trees, autochthonous tree species. All this corresponds to the selective management system that has been applied in forestry in Bosnia and Herzegovina for decades, so that the naturalness of forests in Bosnia and Herzegovina has been largely preserved. Natural forests are $95 \%$ of the total forest area in Bosnia and Herzegovina. Natural forests are not those created by afforestation but those that naturally regenerate (VIŠNJIĆ, 2006). Forests of beech and fir (with spruce) are spread in Bosnia and Herzegovina from mountain to high mountain belt, between 600 and 1,600 meters above sea level. In its geographic distribution across Croatia and Slovenia, beech and fir trees represent the largest forest communities in Europe (BONČINA ET AL., 2003).

In Bosnia and Herzegovina there are remains of untouched-pristine forests of beech and fir (with spruce) such as for example: Perućica, Ravna Vala, Mačen Do, Bobija, Trstionica and Plješevica... (VoJNIKOVIĆ, 2017). The researches of the structural and floristic composition were carried out in some pristine forest reserves (PINTARIĆ, 1978, LeIBUNDGUT 1986, PINTARIĆ, 1991, BEUS AND VoJNIKOVIĆ, 2002, 2005, 2006; MEŠKOVIĆ, 2004; SEBASTIA ET AL., 2005; VIŠNJIĆ ET AL., 2009, 2013 AND 2015). In other areas of South-eastern Europe, the results of numerous researches on the structural composition of pristine forest reserves have been published (LEIBUNDGUT, 1993, KORPEL, 1995, TABAKU, 1999, VRŠKA ET AL., 2001, MEYER ET AL, 2003, ANIĆ ET AL. 2006; DuBRAVEC ET AL., 2006 AND 2007; DIACI ET AL., 2011). In most European countries, silviculture goals are directed towards natural multifunctional forest management. For this type of management there is a lack of comparative reference value from pristine forests, or they are theoretically modelled (MEYER ET AL., 2001). Comparative analyses of pristine forest ecosystems with the managed forests show the impact of humans on forests and signalize the consequences of improper management if it is present, or help defining future activities (DROESSLER, 2006).

By comparing structural composition, tree species composition, competition, diversity and other ecological production parameters in the pristine forest and the managed forest, such reference values can be defined, which is of utmost importance for the optimization of activities in the forests in the function of sustainable management. 


\section{MATERIAL AND METHODS - Materijal i metode}

\section{The object of research}

The research object is located in central Bosnia, about $20 \mathrm{~km}$ southwest of Sarajevo, and covers an area of 45 ha of "Ravna vala" pristine forest as well as about 1500 ha of the managed forests of beech and fir (with spruce) Abieti - Fagetum illyricum Treg. 1957. (BEUS AND VOJNIKOVIĆ, 2002). Experimental plots are located at an altitude from 1280 to $1450 \mathrm{~m}$ and are exposed to the east and northeast. The parent substrate consists of limestone and dolomite as well as moraine deposits on which different depths of the type of rendsina, calcomelanosol, and calcocambisol have developed mosaically on smaller surfaces. (ĆIRIĆ, 1966). The climate is continental with a strong mountainous influence (LUČIĆ, 1966). The mean annual temperature is 6 ${ }^{\circ} \mathrm{C}$, and the total annual rainfall is $1600 \mathrm{~mm}$. Beech and fir dominates the area of research, while the share of spruce is considerably smaller. Fine deciduous trees sycamore and wych elm are individually present.

\section{Research methods}

Experimental plots in the form of concentric circles (five circuits on the plot) were set to determine the structural characteristics of the pristine forest, with the largest radius of the circle of 25 meters and the smallest of $2.5 \mathrm{~m}$, according to the method of STOJANOVIĆ AND DRINIĆ (1975). A square mesh with 100 meters long sides was put in "Ravna vala" pristine forest. The centres of the circular surfaces were put on the intersections of the sides. Belonging to the species, the diameter of trees on the chest height, the height of each tree and the presence and degree of decay of dead wood was determined on concentric circular surfaces. The polar method established the position of each tree in the circle in relation to the centre of the circle. A total of 45 experimental plots were put in the pristine forest.

In the managed forest bordering the pristine forest, experimental plots of a circular shape of radius of 25 meters were put. In order to position the experimental plot in the managed forest, a square mesh with 1000 meters long sides was put. Centres of experimental plots of a circular shape of radius of 25 meters were put at the intersections of the sides, A total of 15 experimental plots were put in the managed forest. On all surfaces, the diameter of all trees above the valuation threshold $(>7 \mathrm{~cm})$ was measured; the height of trees, the belonging to the species was determined, dead wood, as well as the position of each tree above the valuation threshold in relation to the centre of the circle by the polar method.

Dead wood was found on all experimental plots both in the pristine forest and the managed forest. Differentiation was made between the dead wood of deciduous and coniferous trees during the survey. The category of dead wood and the degree of decomposition was determined according to ALBRECHT method (1991). The parts of dead wood thinner than $7 \mathrm{~cm}$ were not covered by measurement.

The stand diversity was determined according to the procedure developed by Jähne and DOHRENBUSCH (1997). The stand diversity index is modularly constructed and 
uses the following parameters for calculation: diversity species, spatial distribution of trees on the surface, vertical stand structure of the tree and the differentiation of tree crowns. Calculation of this index does not require a complete survey that is carried out only in extreme cases. The procedure enables fast coverage of available data for large areas.

Experimental plots of a square shape with a size of $20 \mathrm{~m} \times 20 \mathrm{~m}$, were set according to the BRAUN-BLANQUET (1964) method for the description of the vegetation characteristics,. A total of 13 such plots were put in the managed forest and 8 in the pristine forest. The diversity of the vascular flora is expressed through the ShannonWeaver-Index (Shannon und Weaver 1976) and the Evenness Index. Software applications Turboveg und Juice 4.3.1 (TICHY 2007) were used to process vegetation data. Correspondence Analysis (CA) was carried out using the Canoco 4.5 software (TER BRAAK ET AL., 1999-2002).

\section{RESULTS - Rezultati}

\section{Diameter structure}

In both the pristine forest and the managed forest, according to the digressive curve, the number of trees is decreasing with the increase in diameter, showing the selective structure in both cases (Graph 1). Beech dominates lower diameter classes both in the pristine forest and managed forest. Fir dominates the highest diameter classes both in the pristine forest and in the managed forest. The largest measured diameter in the pristine forest is $145 \mathrm{~cm}$ and $70 \mathrm{~cm}$ in the managed forest.
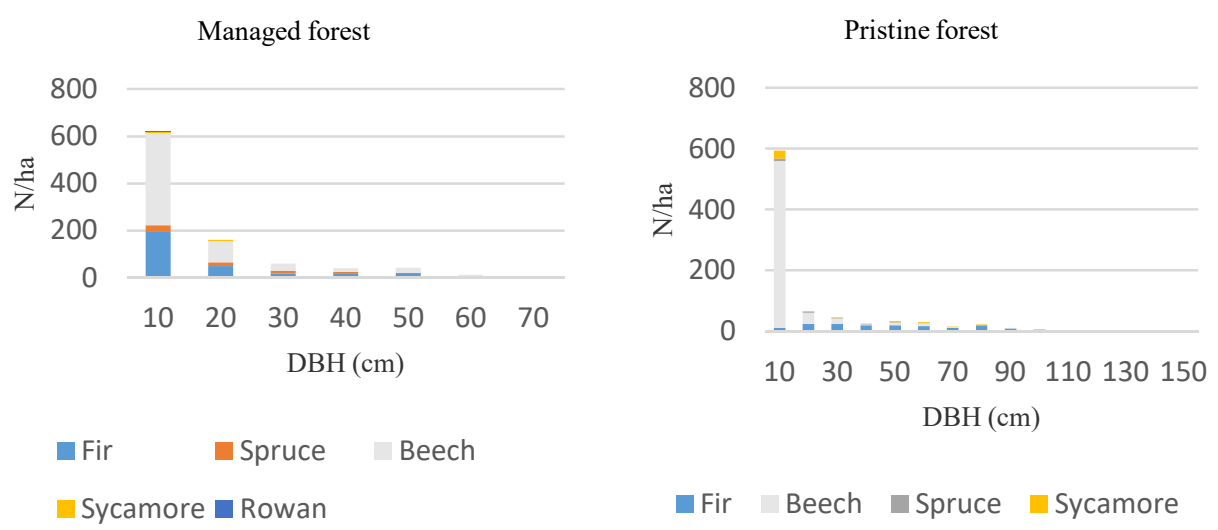

Graphic 1. Frequency distribution of diameter clases in managed forest (left) and pristine forest (right).

Grafikon 1. Distribucija stabala po debljinskim klasama u gospodarskoj šumi (lijevo) $i$ prašumi (desno) 


\section{Volume of live wood of the stands}

Although a total number of trees per hectare are higher in the managed forest, in the pristine forests the basal area and stock of stands are higher (Table 1). In terms of individual tree species, the number of fir trees in the pristine forest is two times smaller than in the managed forest, while the basal area and stock of fir are three times higher in the pristine forest. This can be explained by the fact that fir in the pristine forest is mostly present in higher diameter classes. The beech in the pristine forest has a larger share regarding the number of trees, and also a larger share of the basal area and stock compared to the managed forest, which is the result of a larger number of beech trees in lower diameter classes in the pristine forest. There is a smaller number as well as smaller stock of spruce trees in the pristine forest. It has a similar distribution by diameter classes in pristine forest and the managed forest.

Table 1. Indices of forest yield of live wood stock in Pristine Forest and Managed Forest. Tabela 1. Prinosni pokazatelji živog dijela sastojine u prašumi i gospodarskoj šumi.

\begin{tabular}{|l|c|c|c|c|c|c|}
\hline & \multicolumn{3}{|c|}{ Pristine Forest/prašuma } & \multicolumn{3}{c|}{ Managed Forest/ Gospodarska šuma } \\
\hline $\begin{array}{l}\text { Tree species/ } \\
\text { vrste drveća }\end{array}$ & $\mathrm{N} \mathrm{ha}^{-1}$ & $\mathrm{G} \mathrm{ha}^{-1}$ & $\mathrm{~m}^{3} \mathrm{ha}^{-1}$ & $\mathrm{~N} \mathrm{ha}^{-1}$ & $\mathrm{G} \mathrm{ha}^{-1}$ & $\mathrm{~m}^{3} \mathrm{ha}^{-1}$ \\
\hline Abies alba & 146 & 31,83 & 511,85 & 304 & 11,05 & 144,82 \\
\hline Picea abies & 14 & 1,19 & 17,70 & 73 & 5,05 & 57,19 \\
\hline Fagus sylvatica & 590 & 15,70 & 235,90 & 553 & 14,67 & 157,30 \\
\hline Others/ostale & $1^{1}$ & 0,26 & 5,18 & $14^{2}$ & 0,57 & 7,18 \\
\hline Total/ukupno & 751 & 48,97 & 770,64 & 944 & 31,34 & 366,50 \\
\hline
\end{tabular}

$\left.{ }^{1}\right)$ Acer pseudoplatanus

2) 11 Acer pseudoplatanus, 2 Sorbus aucuparia, 1 Betula pendula

\section{Volume of dead wood}

On average there is $32 \mathrm{~m}^{3} / \mathrm{ha}$ of dead wood in the managed forest, while 167 $\mathrm{m}^{3} /$ ha was recorded in the pristine forest, which is about 5 times more than in the managed forest. (Table 2). The share of dead wood in the total stock of the managed forest is $8 \%$, while the share of dead wood in the total stock of the pristine forest is $18 \%$. If one observes the percentage share of dead wood of coniferous and deciduous trees, it can be seen that both in the managed and the pristine forest, the share of dead wood of coniferous trees is higher than that of dead wood deciduous trees. The percentage share of coniferous trees is lower in the managed than in the pristine forest, but the percentage share of dead wood of deciduous and unclassified trees is higher in the managed forest.

The results of the research show that the degree of decomposition 1 (recently dead wood) is not present in the managed forest, while only a small share is present in the pristine forest $(3.65 \%)$. The degree of decomposition 4 (very decomposed wood) in the managed forest occupies the largest share $(41.25 \%)$. In the pristine forest, in contrast, the deadwood is divided into equal parts according to the degree 
of decomposition 2 (initial decomposition: 38.8\%) and degree of decomposition 3 (advanced decomposition: 39.45\%).

The managed forest and pristine forest also differ in terms of the diameter structure of dead wood. While in the managed forests, there is mostly thinner dead wood with a diameter of 7 to $20 \mathrm{~cm}$, while in the pristine forest there is a dead wood of larger dimensions, mostly consisting of lying and standing dead trees.

Table 2. Overall accumulation of dead wood $\left(\mathrm{m}^{3} \mathrm{ha}^{-1}\right)$ in Managed Forest and Pristine Forest, broken down by wood type and decomposition degree $\left(Z^{\circ}\right), 1$ - just dead, 2 - starting decomposition, 3 - advanced decompostion, 4 - heavily degraded/moldered.

Tabela 2. Sveukupna zaliha mrtvog drveta $\left(m^{3} h a^{-1}\right)$ u gospodarskoj šumi i prašumi, razdijeljena na četinare $i$ lišćare $i$ stepen raspadanja $\left(Z^{\circ}\right)$ sa 1- svježe mrtvo drvo, 2započelo raspadanje, 3-uznapredovalo raspadanje, 4-jako razloženo/molder

\begin{tabular}{|c|c|c|c|c|c|c|c|c|c|c|}
\hline Tree species & \multicolumn{4}{|c|}{ Managed forest/gospodarska šuma } & \multicolumn{5}{c|}{ Pristine forest/Prašuma } \\
\hline & $\mathrm{Z}^{\circ} 1$ & $\mathrm{Z}^{\circ} 2$ & $\mathrm{Z}^{\circ} 3$ & $\mathrm{Z}^{\circ} 4$ & Total & $\mathrm{Z}^{\circ} 1$ & $\mathrm{Z}^{\circ} 2$ & $\mathrm{Z}^{\circ} 3$ & $\mathrm{Z}^{\circ} 4$ & Total \\
\hline Hardwood & 0 & 3,23 & 2,73 & 0,41 & 6,37 & 0,01 & 5,85 & 7,45 & 2,71 & 16,02 \\
\hline Conifer & 0 & 4,51 & 6,11 & 8,23 & 18,85 & 5,98 & 59,00 & 53,90 & 11,71 & 130,59 \\
\hline Unknown & 0 & 0 & 2,10 & 4,44 & 6,54 & 0 & 0 & 4,53 & 16,20 & 20,73 \\
\hline Total & 0 & 7,74 & 10,94 & 13,08 & 31,75 & 5,99 & 64,85 & 65,88 & 30,62 & 167,34 \\
\hline
\end{tabular}

\section{Diversity}

Table 3 shows the diversity of trees in the pristine forest and managed forest regarding the number of trees and basal area per hectare, as well as the stand diversity according to JÄHNE AND DOHRENBUSCH (1997). The managed forest shows higher diversity in comparison with the pristine forest, and over the number of trees and through basal area. The evenness index is also higher in the managed forest compared to the pristine forest.

Unlike the previous, the stand diversity index is higher in the pristine forest. It is clear that the pristine forest shows the higher ecological and structural diversity of the managed forest when multiple parameters of diversity are taken into account. However, it should be emphasized that the stand diversity index in the pristine forest and the managed forest do not differ significantly (pristine forest 8.8, managed forest 8.3 ), which is an indicator of a high stand diversity both in the managed and pristine forests. 
Table 3.Comparison of stand diversity of Managed Forest and Pristine Forest.

Tabela 3. Uporedba diverzteta vaskularne flore $i$ sastojinskog diveziteta u prašumi $i$ gospodarskoj šumi.

\begin{tabular}{|c|c|c|c|c|c|}
\hline & \multicolumn{2}{|c|}{ SHANNON-Index } & \multicolumn{2}{c|}{ Evenness } & \multirow{2}{*}{$\begin{array}{c}\text { Stand diversity } \\
\text { index }\end{array}$} \\
\hline & $\mathrm{N} \mathrm{ha}^{-1}$ & $\mathrm{G}\left(\mathrm{m}^{2}\right)$ & $\mathrm{N} \mathrm{ha}^{-1}$ & $\mathrm{G}\left(\mathrm{m}^{2}\right)$ & 8,8 \\
\hline Pristine forest & 0,588 & 0,7629 & 0,424 & 0,5503 & 8,3 \\
\hline Managed forest & 0,937 & 1,089 & 0,676 & 0,7862 & \\
\hline
\end{tabular}

\section{Vegetation characteristics}

Table 4 shows the species of bush trees and ground vegetation registered in the pristine forest and managed forest. In the layer of trees, dominant are fir (Abies alba), beech (Fagus sylvatica) and spruce (Picea abies). In addition to these, sycamore (Acer Pseudoplatanus) is also found in the pristine forest. In the layer of shrubs honeyberry is found: Lonicera xylosteum, L. alpigena and L. nigra. In the managed forests, there is also the Corylus avellana and typical Illyrian Balkan species of buckthorn Rhamnus Alpinus s. fallax. In the layer of ground vegetation Festuca altissima dominates, while the representation in the managed forest (degree of coverage 5) is somewhat higher than in the pristine forest (degree of coverage 4$5)$.

The Illyrian species Cardamine waldsteinii, Euphorbia carniolica, Aremonia agrimonoides characterize this forest community as Abieti-Fagetum illyricum Treg. 1957 (nom. Illeg.) in relation to Aremonio-Fagion (HORVAT 1938; BORHIDI AND TÖRÖK, PODANI AND BORHIDI 1989).

A total of 60 plant species were recorded in the pristine forest. There are 87 of them in the managed forest. There are 44 species of plant species found in the managed forest and the pristine forest. Of the plant species registered only in the managed forest, there are some pioneer species from order of Adenostyletalia, as well as anthropogenic species: Salix caprea, Populus tremula, Corylus avellana, Sambucus ebulus, Aconitum lycoctonum, Atropa bella-donna, Carduus personata, Digitalis grandiflora, Doronicum columnae, Geranium phaeum, Heracleum sphondylium, Lamium maculatum, Ranunculus platanifolius, Stellaria nemorum, Telekia speciosa, Thalictrum aquilegiifolium, Urtica dioica. Species recorded only in the pristine forests are: Fraxinus excelsior, Carex pendula, Cicerbita alpina and Melica uniflora.

Table 4. Recorded plant species in Managed forest (13 rélevés) and Pristine Forest (8 rélevés);

Forest layer: 1 = Overstorey, 2 = Understorey, 4-5 = Shrubs and Herbs, $6=$ Ground Vegetation; $\mathrm{DG}^{\circ}(\%)$ - Percentage Constancy; DG (BB) - Braun-Blanquet cover range.

Tabela 4. Registrovane biljne vrste u gospodarskoj šumi (13 snimaka) i prašumi (8 snimaka); Slojevi: 1-prvi sprat drveća, 2- drugi sprat drveća, 4-5-sprat grmlja, 6-prizemna vegetacja; $D G^{\circ}(\%)$ stepen prisutnosti, DG 8BB)- stepen pokrovnosti po Braun Blanquet 
Stand structure, dead wood and floristic composition of a pristine forest stand of beech and fir compared to a managed forest in the Dinaric mountain Bjelašnica

\begin{tabular}{|c|c|c|c|c|c|c|c|}
\hline \multicolumn{4}{|c|}{ Managed Forest/Gospodarska šuma } & \multicolumn{4}{|c|}{ Pristine Forest/Prašuma } \\
\hline Plant species & $\begin{array}{l}\text { (Forest } \\
\text { Layer) }\end{array}$ & $\begin{array}{l}\mathrm{DG}^{\circ} \\
(\%)\end{array}$ & $\begin{array}{l}\mathrm{DG} \\
\text { (BB) }\end{array}$ & Plant species & $\begin{array}{l}\text { (Forest } \\
\text { Layer) }\end{array}$ & $\begin{array}{l}\mathrm{DG}^{\circ} \\
(\%)\end{array}$ & $\begin{array}{l}\mathrm{DG} \\
\text { (BB) }\end{array}$ \\
\hline Abies alba & 1 & 54 & $1-4$ & Abies alba & 1 & 100 & $2-4$ \\
\hline Fagus sylvatica & 1 & 77 & $1-4$ & $\begin{array}{c}\text { Acer } \\
\text { pseudoplatanus }\end{array}$ & 1 & 25 & 1 \\
\hline Picea abies & 1 & 85 & $1-3$ & Fagus sylvatica & 1 & 88 & $2-4$ \\
\hline Abies alba & 2 & 46 & $1-2$ & Picea abies & 1 & 12 & 2 \\
\hline $\begin{array}{c}\text { Acer } \\
\text { pseudoplatanus }\end{array}$ & 2 & 8 & 1 & Abies alba & 2 & 25 & $1-2$ \\
\hline Fagus sylvatica & 2 & 62 & $1-5$ & $\begin{array}{c}\text { Acer } \\
\text { pseudoplatanus }\end{array}$ & 2 & 12 & 1 \\
\hline Picea abies & 2 & 69 & $1-2$ & Fagus sylvatica & 2 & 100 & $2-4$ \\
\hline Salix caprea & 2 & 8 & 1 & Picea abies & 2 & 12 & 1 \\
\hline Sorbus aucuparia & 2 & 8 & 2 & Fagus sylvatica & 4 & 25 & +-1 \\
\hline Ulmus glabra & 2 & 8 & 1 & Abies alba & 5 & 12 & + \\
\hline Picea abies & 4 & 8 & + & $\begin{array}{c}\text { Acer } \\
\text { pseudoplatanus }\end{array}$ & 5 & 38 & +-1 \\
\hline Abies alba & 5 & 85 & +-4 & Fagus sylvatica & 5 & 25 & $1-3$ \\
\hline $\begin{array}{c}\text { Acer } \\
\text { pseudoplatanus }\end{array}$ & 5 & 69 & +-2 & Fraxinus excelsior & 5 & 12 & + \\
\hline Corylus avellana & 5 & 8 & + & Lonicera alpigena & 5 & 25 & 1 \\
\hline Daphne mezereum & 5 & 15 & + & Lonicera nigra & 5 & 25 & +-1 \\
\hline Fagus sylvatica & 5 & 85 & $1-4$ & Picea abies & 5 & 12 & 1 \\
\hline Lonicera alpigena & 5 & 8 & 1 & $\begin{array}{l}\text { Rhammus alpinus } s . \\
\text { fallax }\end{array}$ & 5 & 62 & +-3 \\
\hline Lonicera nigra & 5 & 15 & +-1 & Sorbus aucuparia & 5 & 50 & +-1 \\
\hline $\begin{array}{c}\text { Lonicera } \\
\text { xylosteum }\end{array}$ & 5 & 15 & +-1 & $\begin{array}{c}\text { Acer } \\
\text { pseudoplatanus }\end{array}$ & 6 & 75 & +-1 \\
\hline Picea abies & 5 & 69 & +-2 & $\begin{array}{c}\text { Adenostyles } \\
\text { alliariae }\end{array}$ & 6 & 75 & +-1 \\
\hline Populus tremula & 5 & 8 & + & $\begin{array}{c}\text { Aegopodium } \\
\text { podagraria }\end{array}$ & 6 & 75 & +-1 \\
\hline $\begin{array}{l}\text { Rhamnus alpinus } s . \\
\text { fallax }\end{array}$ & 5 & 62 & $1-3$ & Ajuga reptans & 6 & 100 & +-1 \\
\hline Rubus idaeus & 5 & 23 & +-1 & $\begin{array}{l}\text { Anemone } \\
\text { nemorosa }\end{array}$ & 6 & 62 & +-1 \\
\hline Sambucus ebulus & 5 & 8 & + & $\begin{array}{c}\text { Aremonia } \\
\text { agrimonoides }\end{array}$ & 6 & 100 & +-1 \\
\hline Samucus racemosa & 5 & 8 & + & $\begin{array}{c}\text { Asarum } \\
\text { europaeum }\end{array}$ & 6 & 50 & +-1 \\
\hline Sorbus aucuparia & 5 & 54 & +-2 & $\begin{array}{l}\text { Athyrium filix- } \\
\text { femina }\end{array}$ & 6 & 88 & +-1 \\
\hline
\end{tabular}




\begin{tabular}{|c|c|c|c|c|c|c|c|}
\hline $\begin{array}{c}\text { Acer } \\
\text { pseudoplatanus }\end{array}$ & 6 & 15 & +-1 & $\begin{array}{l}\text { Cardamine } \\
\text { bulbifera }\end{array}$ & 6 & 100 & + \\
\hline $\begin{array}{l}\text { Aconitum } \\
\text { lycoctonum }\end{array}$ & 6 & 15 & +-1 & $\begin{array}{l}\text { Cardamine } \\
\text { enneaphyllos }\end{array}$ & 6 & 100 & +-1 \\
\hline Actaea spicata & 6 & 46 & + & Carex digitata & 6 & 12 & + \\
\hline $\begin{array}{c}\text { Adenostyles } \\
\text { alliariae }\end{array}$ & 6 & 8 & + & Carex pendula & 6 & 12 & + \\
\hline $\begin{array}{l}\text { Aegopodium } \\
\text { podagraria }\end{array}$ & 6 & 23 & + & Carex sylvatica & 6 & 50 & + \\
\hline Ajuga reptans & 6 & 54 & +-1 & Cicerbita alpina & 6 & 12 & + \\
\hline $\begin{array}{l}\text { Anemone } \\
\text { nemorosa }\end{array}$ & 6 & 8 & + & Corylus avellana & 6 & 25 & + \\
\hline Angelica sylvestris & 6 & 8 & + & $\begin{array}{l}\text { Cystopteris } \\
\text { montana }\end{array}$ & 6 & 38 & + \\
\hline $\begin{array}{c}\text { Aremonia } \\
\text { agrimonoides }\end{array}$ & 6 & 77 & +-1 & $\begin{array}{l}\text { Dactylorhiza } \\
\text { fuchsii }\end{array}$ & 6 & 12 & + \\
\hline $\begin{array}{c}\text { Asarum } \\
\text { eruopaeum }\end{array}$ & 6 & 62 & +-1 & $\begin{array}{l}\text { Dryopteris filix- } \\
\text { mas }\end{array}$ & 6 & 100 & +-1 \\
\hline $\begin{array}{l}\text { Athyrium filix- } \\
\text { femina }\end{array}$ & 6 & 46 & +-1 & $\begin{array}{c}\text { Euphorbia } \\
\text { amygdaloides }\end{array}$ & 6 & 75 & + \\
\hline $\begin{array}{l}\text { Atropa bella- } \\
\text { donna }\end{array}$ & 6 & 8 & + & $\begin{array}{l}\text { Euphorbia } \\
\text { carniolica }\end{array}$ & 6 & 75 & + \\
\hline $\begin{array}{l}\text { Brachypodium } \\
\text { sylvaticum }\end{array}$ & 6 & 8 & 1 & Festuca altissima & 6 & 100 & $4-5$ \\
\hline $\begin{array}{l}\text { Cardamine } \\
\text { enneaphyllos }\end{array}$ & 6 & 15 & + & Galium odoratum & 6 & 100 & +-1 \\
\hline $\begin{array}{l}\text { Cardamine } \\
\text { waldsteinii }\end{array}$ & 6 & 23 & + & Galium sylvaticum & 6 & 38 & + \\
\hline Carduus personata & 6 & 15 & + & $\begin{array}{c}\text { Gentiana } \\
\text { asclepiadea }\end{array}$ & 6 & 75 & + \\
\hline Cares digitata & 6 & 31 & + & $\begin{array}{c}\text { Geranium } \\
\text { robertianum }\end{array}$ & 6 & 25 & + \\
\hline Carex sylvatica & 6 & 31 & + & $\begin{array}{l}\text { Hordelymus } \\
\text { europaeus }\end{array}$ & 6 & 62 & + \\
\hline Corylus avallana & 6 & 8 & + & $\begin{array}{c}\text { Lamium } \\
\text { galeobdolon }\end{array}$ & 6 & 75 & +-1 \\
\hline $\begin{array}{l}\text { Cystopteris } \\
\text { montana }\end{array}$ & 6 & 15 & + & Laserpitium krapfii & 6 & 88 & + \\
\hline Daphne mezereum & 6 & 15 & + & Lilium martagon & 6 & 100 & + \\
\hline $\begin{array}{l}\text { Digitalis } \\
\text { grandiflora }\end{array}$ & 6 & 8 & + & Lonicera alpigena & 6 & 62 & +-1 \\
\hline $\begin{array}{l}\text { Doronicum } \\
\text { columnae }\end{array}$ & 6 & 31 & + & Lonicera nigra & 6 & 12 & 1 \\
\hline $\begin{array}{c}\text { Dryopteris filix- } \\
\text { mas }\end{array}$ & 6 & 85 & +-4 & $\begin{array}{l}\text { Lonicera } \\
\text { xylosteum }\end{array}$ & 6 & 12 & + \\
\hline $\begin{array}{l}\text { Epilobium } \\
\text { montanum }\end{array}$ & 6 & 31 & + & Melica uniflora & 6 & 12 & + \\
\hline
\end{tabular}


Stand structure, dead wood and floristic composition of a pristine forest stand of beech and fir compared to a managed forest in the Dinaric mountain Bjelašnica

\begin{tabular}{|c|c|c|c|c|c|c|c|}
\hline $\begin{array}{c}\text { Euphorbia } \\
\text { amygdaloides }\end{array}$ & 6 & 62 & +-1 & Mycelis muralis & 6 & 38 & + \\
\hline $\begin{array}{l}\text { Euphorbia } \\
\text { carniolica }\end{array}$ & 6 & 23 & + & Neottia nidus-avis & 6 & 62 & + \\
\hline Festuca altissima & 6 & 85 & +-5 & Oxalis acetosella & 6 & 88 & + \\
\hline $\begin{array}{c}\text { Festuca } \\
\text { heterophylla }\end{array}$ & 6 & 15 & +-1 & Paris quadrifolia & 6 & 50 & + \\
\hline Fragaria vesca & 6 & 31 & + & $\begin{array}{l}\text { Phyteuma } \\
\text { spicatum }\end{array}$ & 6 & 50 & + \\
\hline Galium odoratum & 6 & 85 & +-2 & Picea abies & 6 & 12 & + \\
\hline Galium sylvaticum & 6 & 31 & +-1 & $\begin{array}{l}\text { Polygonatum } \\
\text { verticillatum }\end{array}$ & 6 & 12 & + \\
\hline $\begin{array}{c}\text { Gentiana } \\
\text { asclepiadea }\end{array}$ & 6 & 23 & +-1 & $\begin{array}{l}\text { Polystichum } \\
\text { setiferum }\end{array}$ & 6 & 12 & + \\
\hline Geranium phaeum & 6 & 15 & + & $\begin{array}{l}\text { Prenanthes } \\
\text { purpurea }\end{array}$ & 6 & 88 & +-1 \\
\hline $\begin{array}{c}\text { Geranium } \\
\text { robertianum }\end{array}$ & 6 & 31 & + & $\begin{array}{l}\text { Pulmonaria } \\
\text { officinalis }\end{array}$ & 6 & 12 & + \\
\hline $\begin{array}{c}\text { Heracleum } \\
\text { sphondylium }\end{array}$ & 6 & 23 & + & $\begin{array}{l}\text { Rhamnus alpinus } s \text {. } \\
\text { fallax }\end{array}$ & 6 & 38 & +-1 \\
\hline $\begin{array}{l}\text { Hordelymus } \\
\text { europaeus }\end{array}$ & 6 & 38 & + & Rosa pendulina & 6 & 62 & + \\
\hline $\begin{array}{l}\text { Hypericon } \\
\text { montanum }\end{array}$ & 6 & 15 & + & Rubus hirtus & 6 & 12 & + \\
\hline Knautia dinarica & 6 & 23 & + & Salvia glutinosa & 6 & 62 & +-1 \\
\hline $\begin{array}{c}\text { Lamium } \\
\text { galeobdolon }\end{array}$ & 6 & 85 & +-2 & Sanicula europaea & 6 & 100 & 1 \\
\hline $\begin{array}{l}\text { Lamium } \\
\text { maculatum }\end{array}$ & 6 & 8 & 1 & $\begin{array}{c}\text { Saxifraga } \\
\text { rotundifolia }\end{array}$ & 6 & 12 & + \\
\hline Laserpitium krapfii & 6 & 38 & + & $\begin{array}{c}\text { Senecio } \\
\text { germanicus }\end{array}$ & 6 & 100 & + \\
\hline Lathyrus vernus & 6 & 8 & + & Sorbus aucuparia & 6 & 50 & + \\
\hline Lilium martagon & 6 & 15 & + & $\begin{array}{l}\text { Symphytum } \\
\text { tuberosum }\end{array}$ & 6 & 50 & +-1 \\
\hline Lonicera alpigena & 6 & 46 & +-1 & $\begin{array}{l}\text { Vaccinium } \\
\text { myrtillus }\end{array}$ & 6 & 12 & + \\
\hline Lonicera nigra & 6 & 8 & + & Verbascum chaixii & 6 & 12 & + \\
\hline Lunaria rediviva & 6 & 8 & 1 & $\begin{array}{c}\text { Veronica } \\
\text { chamaedrys }\end{array}$ & 6 & 50 & + \\
\hline Luzula sylvatica & 6 & 31 & +-1 & $\begin{array}{l}\text { Veronica } \\
\text { urticifolia }\end{array}$ & 6 & 25 & + \\
\hline $\begin{array}{l}\text { Melampyrum } \\
\text { sylvaticum }\end{array}$ & 6 & 15 & + & $\begin{array}{c}\text { Viola } \\
\text { reichenbachiana }\end{array}$ & 6 & 100 & +-1 \\
\hline Milium effusum & 6 & 15 & + & & & & \\
\hline Mycelis muralis & 6 & 38 & +-1 & & & & \\
\hline Myosotis sylvatica & 6 & 15 & + & & & & \\
\hline
\end{tabular}




\begin{tabular}{|c|c|c|c|c|c|c|c|}
\hline Oxalis acetosella & 6 & 62 & +-2 & & & & \\
\hline Paris quadrifolia & 6 & 31 & + & & & & \\
\hline $\begin{array}{l}\text { Phyteuma } \\
\text { spicatum }\end{array}$ & 6 & 8 & + & & & & \\
\hline Picea abies & 6 & 8 & + & & & & \\
\hline $\begin{array}{l}\text { Polygonatum } \\
\text { verticillatum }\end{array}$ & 6 & 38 & + & & & & \\
\hline $\begin{array}{l}\text { Polystichum } \\
\text { setiferum }\end{array}$ & 6 & 54 & + & & & & \\
\hline $\begin{array}{l}\text { Prenanthes } \\
\text { purpurea }\end{array}$ & 6 & 69 & + & & & & \\
\hline $\begin{array}{l}\text { Pulmonaria } \\
\text { officinalis }\end{array}$ & 6 & 8 & + & & & & \\
\hline $\begin{array}{l}\text { Ranunculus } \\
\text { platanifolius }\end{array}$ & 6 & 8 & + & & & & \\
\hline $\begin{array}{l}\text { Rhamnus alpinus } s . \\
\text { fallax }\end{array}$ & 6 & 8 & + & & & & \\
\hline Rosa pendulina & 6 & 46 & + & & & & \\
\hline Rubus hirtus & 6 & 15 & + & & & & \\
\hline Rubus idaeus & 6 & 38 & +-2 & & & & \\
\hline $\begin{array}{l}\text { Sambucus } \\
\text { racemosa }\end{array}$ & 6 & 8 & + & & & & \\
\hline Sanicula europaea & 6 & 46 & + & & & & \\
\hline $\begin{array}{c}\text { Saxifraga } \\
\text { rotundifolia }\end{array}$ & 6 & 23 & + & & & & \\
\hline $\begin{array}{l}\text { Scrophularia } \\
\text { nodosa }\end{array}$ & 6 & 15 & + & & & & \\
\hline $\begin{array}{c}\text { Senecio } \\
\text { germanicus }\end{array}$ & 6 & 31 & + & & & & \\
\hline Stellaria nemorum & 6 & 8 & 1 & & & & \\
\hline $\begin{array}{c}\text { Symphytum } \\
\text { tuberosum }\end{array}$ & 6 & 31 & + & & & & \\
\hline Telekia speciosa & 6 & 8 & + & & & & \\
\hline $\begin{array}{c}\text { Thalictrum } \\
\text { aquilegifolium }\end{array}$ & 6 & 8 & + & & & & \\
\hline Urtica dioica & 6 & 23 & +-4 & & & & \\
\hline $\begin{array}{l}\text { Vaccinium } \\
\text { myrtillus }\end{array}$ & 6 & 38 & +-3 & & & & \\
\hline $\begin{array}{c}\text { Veronica } \\
\text { chamaedrys }\end{array}$ & 6 & 23 & + & & & & \\
\hline $\begin{array}{l}\text { Veronica } \\
\text { urticifolia }\end{array}$ & 6 & 23 & + & & & & \\
\hline $\begin{array}{c}\text { Viola } \\
\text { reichenbachiana }\end{array}$ & 6 & 85 & +-1 & & & & \\
\hline
\end{tabular}

Despite the large number of plant species identified in the managed forest, when comparing the diversity index, no statistically significant difference was found 
in the diversity indexes of vascular flora in the managed forest and the pristine forest (Table 3).

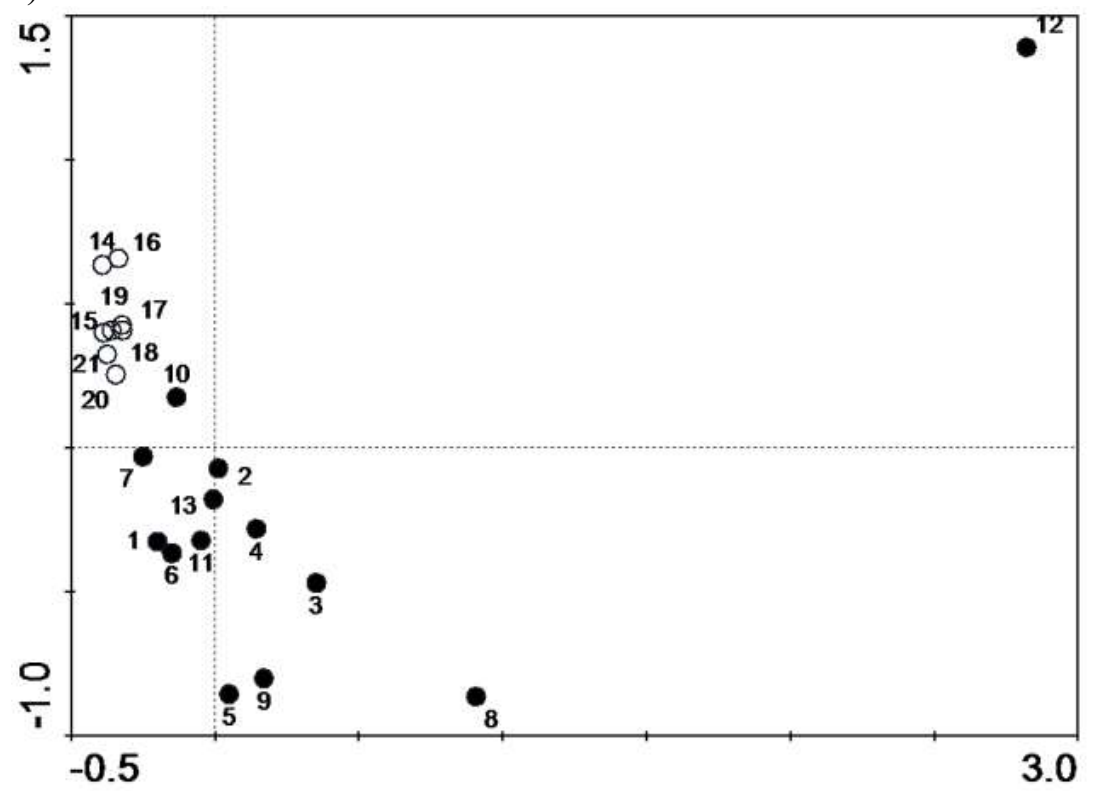

Graphic 2. Correspondence Analysis of floristic composition (•Managed Forest: Plots 1-13; ○ Pristine Forest: Plots 14-21).

Grafikon 2. Korespodentna analiza sa florstičkim sastavom (•Gospodarska šuma: Plohe 113; ○ Prašuma: Plohe 14-21).

From the corresponding analysis it can be seen to what extent the pristine forest and the managed forests differ in terms of floristic composition. Graphic 2 show the results of this analysis. Points or circles on the graph represent one experimental plot with its floristic composition. If the points are closer to each other, the floral composition on the plot is more similar and vice versa, if they are more distant the floristic composition between the plots is more different.

The experimental plots from the pristine forest and the managed forest show a clear boundary of separation. The scattering points of the experimental plots from the pristine forest are concentrated along the y axis. Points marking the plots from the pristine forest are located on the upper part, and from the managed forest at the bottom part of the graph. Thus, the y axis can be interpreted as the forest management gradient. It is noticeable that the experimental plots from the pristine forest are closer to each other than those from the managed forest. Therefore, the vegetation of the pristine forest is more homogeneous, while the floristic composition of the managed forest is more variable (Graphic 2). 


\section{DISCUSION - Diskusija}

\section{Structure of the stand}

Distribution of trees by diameter classes, both in the pristine forests and in the managed forests has a declining shape of a curve typical of the selective stands. Similar distributions of diameter classes were obtained in numerous researches conducted within the Dinaric managed forests of beech and fir (with spruce) and pristine forests (PINTARIĆ, 1978; PRPIĆ, 1979; LEIBUNDGUT, 1993; PRPIĆ ET AL., 1995; DIACI ET AL., 2008; 2011; ANIĆ AND MIKAC, 2008; VIŠNJIĆ ET AL., 2009, 2013 AND 2015).

Unlike the pristine forest in which there are trees of breast diameter of $145 \mathrm{~cm}$ in the managed forest, the thickest tree has a diameter of $70 \mathrm{~cm}$. This final diameter in the managed forest is defined by the technical goals of management in the selective forests (PINTARIĆ, 1991).

Compared to other pristine forests of beech and fir (with spruce), the number of trees of 751 per hectare is relatively high. Beech dominates in the total number of trees with a share of about 79\%. VIŠNJIĆ ET AL., (2009) registered 652 trees per hectare in the "Plješevica" pristine forest, where beech also dominates with $64 \%$. ANIĆ ET AL., (2006) found in the "Ćorkova uvala" (Croatia) pristine forest an average of 440 trees per hectare where beech has a share of $49 \%$ and fir of $46 \%$. In all the above researches, a high share of beech was found in the total number of trees, especially in lower diameter classes, while fir was mostly represented in higher diameter classes. This phenomenon indicates the process of suppression of fir in favour of beech from the Dinaric beech and fir forests (ANIĆ ET AL., 2006; DIACI ET AL., 2008 AND 2011; VIŠNJIĆ ET AL., 2013 AND 2015).

In the "Ravna vala" pristine forest, a stock of live wood of $770 \mathrm{~m}^{3} / \mathrm{ha}$ was identified, which is similar to the other Dinaric pristine forests of beech and fir. The stock of $772 \mathrm{~m}^{3} / \mathrm{ha}$ was found in the Plješevica pristine forest (VIŠNJIĆ ET AL., 2009), $671 \mathrm{~m}^{3} / \mathrm{ha}$ in Croatia (ANIĆ AND MIKAC, 2008) or $800 \mathrm{~m}^{3} / \mathrm{ha}$ in Slovenia DIACI ET AL. (2008).

The stock in the managed forest with an average of $366 \mathrm{~m}^{3} / \mathrm{ha}$ is two times smaller than in the pristine forest. According to KORPEL (1995), optimal stock in managed forests of beech and fir should be between 250 and $450 \mathrm{~m} 3 / \mathrm{ha}$. KORPEL (1995) suggests that, when transferring the pristine forests of beech and fir to the managed forest, the initial stock should be reduced by more than half. This paper has also established that the reserves of the stands in the part being managed have reduced by half by economic activities compared by the pristine forest.

\section{Volume of dead wood}

Dead wood is a very important structural element in sustainable "close-tonature" forest management. It plays a very important role and represents a key element in preserving the biodiversity of forest ecosystems. Dead wood is important for the development of many organisms, for example, mushrooms, moss and lichens, arthropods and birds. The researches of dead wood in the pristine forests across 
Europe have shown that the volume of dead wood ranges between 50 and $200 \mathrm{~m}^{3} / \mathrm{ha}$, or that the share of dead wood is from 10 to $35 \%$ in the total volume of stands (Albrecht, 1991; LeibundGut, 1993, KorPel, 1995; ChRISTENSEN ET AL., 2005, DEBELJAK, 2006; DIACI ET AL., 2011. VIŠNJIĆ ET AL., 2009 AND 2013). In the "Ravna vala" pristine forest reserve, the supply of dead wood is $167 \mathrm{~m}^{3} /$ ha or $18 \%$ of the total volume (dead and live wood) and it is within the above-mentioned frames.

According to the state forest inventory in Germany (2003), the average volume of dead wood was $11.5 \mathrm{~m}^{3} /$ ha in the managed forests. In the managed forest, bordering "Ravna Vala" pristine forest reserve, a total of $32 \mathrm{~m}^{3} / \mathrm{ha}$ of dead wood volume has been evidenced by these researches, which is on average three times more than in the German forests. This amount of dead wood in the managed exceeds the value of the optimal amount of deadwood stock in the managed forests recommended by AMMER (1991) and ranges between 15 and $30 \mathrm{~m}^{3} / \mathrm{ha}$, but is below the value given by MüLLER for the managed forests of beech (2005) which is $40 \mathrm{~m}^{3} / \mathrm{ha}$. Deadwood is of great importance for the preservation of the biodiversity of forest ecosystems and its presence is the basis for the conservation of many endangered and protected plant species. Managed forests of beech and fir in Bosnia and Herzegovina have sufficient amounts of dead wood per unit area, which makes them more stable and biologically diverse, which are the basic prerequisites for sustainable, environment-friendly management.

\section{Stand diversity}

The diversity of trees by the number of trees of some species of trees represented in the stands is higher in the managed forests than in the pristine forest. This can be explained by the selective management in the managed forest, which has a function to optimize the composition of stands according to the species of trees and which facilitates the development of less competitive indigenous species of trees. The diversity of trees by basal area of some species of trees represented in the pristine forest and the managed forest is higher than the diversity by number of trees in both the pristine forest and the managed forest. This can be explained by more even distribution of the basal area of main species of trees in the managed forest and pristine forest unlike in the case with number of trees. Fir is represented with a smaller number of individuals, but in higher diameter classes, the basal area of fir is almost equal to basal area of beech with a significantly larger number of trees, but is mostly the same in lower diameter classes. The small value of the evenness index can be explained by an uneven distribution of the number of trees and basal area of certain tree species represented in the researched area. Differences in the diversity can be explained by the fact that only two species of trees in the pristine forest have a major role in the development of the stands while in the managed forest, in addition to beech and fir, spruce can be found as the main species.

Based on the calculated stand diversity according to JÄHNE AND DOHRENBUSCH, (1997), it is evident that the pristine forest and the managed forest have highly structurally varied stands. Contrary to the Shanon's diversity index and the evenness 
index, this stand diversity index has higher values in the pristine forest than in the managed forest.

\section{Floristic composition}

Comparison of the floral composition of the vascular flora in the "Ravna vala" pristine forest and the and the managed forest located in the immediate vicinity shows clear differences in the number of species, as well as insignificant differences compared to the index of diversity. These results coincide with the results of BEUS AND VOJNIKOVIĆ (2002). A number of species of vascular flora in the managed forest are explained by the appearance of certain pioneering species of the order Adenostyletalia as well as anthropogenic species introduced, usually occurring after cutting of intense intensity.

The diversity of vascular flora according to Shannon is 2.47 both in the managed forest and pristine forest, while the evenness index is slightly higher in the managed forest is than the pristine forest. Similar results were obtained by SEBASTIA ET AL., (2005) and BEUS AND VOJNIKOVIĆ, (2006). These similarities can also be explained by the method of calculating the diversity which uses the extent of their coverage, in addition to the number of species present on a particular surface,

Correspondence analysis shows that forms of scattering of points which represent a vascular flora from the pristine forest and managed forest are clearly separated. Phytocenological recordings from the managed forest are much more variable than those from the pristine forest. It can be explained by anthropogenic activities in the forest. Through selective cutting, disorders appear inside the stand. The amount of light is increased at the micro level, which also significantly affects the humus decomposition, the surface temperature of the soil and the water content in the soil. The changed micro conditions can facilitate the development of species with certain ecological requirements for light and water, and which did not have the opportunity for their development before cutting.

\section{CONCLUSIONS - Zaključci}

The research was carried out in "Ravna Vala" pristine forest reserve and the managed forest of beech and fir with spruce bordering the pristine forest. The researches have shown a similar declining distribution of trees by diameter classes in both the pristine forest and the managed forest, with the final diameter in the pristine forest being considerably higher. Both the pristine forest and the managed forest show a typical selective structure. In both stands (pristine forest and managed forest), the dominance of beech is determined especially in lower diameter classes, while the presence of spruce both in pristine and managed forests is very small. On the basis of the above, it can be concluded that in the forests of beech and fir (with spruce) there is a gradual change of species, i.e. the suppression of fir in favour of beech, which will have a primary role in the future in the formation of the stands. The diversity of trees is higher in the managed forest in relation to the pristine forest both by number of trees 
and by basal area of the presented species of trees, while the stand diversity index is higher in the pristine forest, which indicates the need to record more structural parameters of the stand in the calculation of diversity. Dead wood is present in the pristine forest in the amount of $167 \mathrm{~m}^{3} / \mathrm{ha}$ and in the commercial forest $32 \mathrm{~m}^{3} / \mathrm{ha}$. The categories of dead wood and the degree of decomposition are different in the pristine forest and the managed forest. This condition of dead wood in the forest is largely the result of anthropogenic economic activity. There is enough dead wood in the managed forest to preserve the biodiversity of these forests. A few species of vascular flora have been identified in the pristine forest compared to the managed forest. In the managed forest there are some pioneering plant species found in the parts of forests under the influence of man. The number of trees is higher in the managed forest due to economic activities, i.e. the opening of the assembly while cutting trees, which results in the creation of a microhabitat for species less competitive than fir and especially beech.

\section{REFERENCES - Literatura}

ANIĆ, I., MIKAC, S., ORŠANIĆ, M., DRVodelić, D. 2006: Structural relations between pristine and management beech-fir stands (Omphalodo-Fagetum Marinček et al. 1992 ) in forests of the Croatians Dinaric Karst. Periodicum Biologorum, 108 (6), 663-669.

ANIĆ, I., MIKAC, S. 2008: Strukur, Textur und Verjüngung im dinarischen BuchenTannen-Urwald „Ćorkova Uvala“, Forstblätter Nr. 11-12, 505-515.

Albrecht, L. 1991: Die Bedeutung des toten Holzes im Wald. Forstw. Cbl. 110, 106113

AMMER, U. 1991: Konsequenzen aus den Ergebnissen der Totholzforschung für die forstliche Praxis. Forstw. Cbl. 110, 149-157.

BEUS, V., VoJNIKOVIĆ, S. 2002: Floristical characteristics of the pristine forest of beech and fir in Ravna Vala on mountain Bjelašnica. Razprave. IV, Razreda SAZU. XLIII, Ljubljana, 63-78.

BEUS, V., VoJNIKOVIĆ, S. 2005: Floristički sastav prašume i gospodarske šume u Ravnoj Vali na planini Bjelašnici; Radovi Šumraskog fakulteta Univerziteta u Sarajevu; Knj. XXXV, br 1; Sarajevo, str. 25-32.

BEUS, V., VoJNIKOVIĆ, S. 2006: Floristische Diversität von bewirtschafteten Beständen und dem Urwald „Ravna Vala“ im Bjelašnica-Gebirge. In: Tagungsband der Konferenz "Management von Waldökosystemen in Nationalparks und anderen geschützen Gebietens", Jahorina, 55-66.

BONČINA, A., GAŠPERŠIC, F., DIACI, J. 2003: Long-term changes in tree species composition in the Dinaric mountain forests of Slovenia. The Forestry Chronicle 79 (2), 227-232

Braun-Blanquet, J. 1964: Pflanzensoziologie. 3. Ed. Berlin, Wien, New York.

ĆIRIĆ, M. 1966: Soils of the mountain region Igman - Bjelašnica. Rad Šum.fak. i Inst. za šum. u Sarajevu 10 (1), 5-44 
Christensen, M., Hahn, K., Mountford, P. E., Oder, P., Standovar, T., RožEnBerger, D., DIACI, J., WIJDEVEN, S., MEYER, P., WINTER, S., VRŠKA, T. 2005: Dead wood in European beech (Fagus sylvatica) forest reserves. For.Ecol.Manag. 210, 267-282.

Diacl, J., Roženbergar, D., Mikac, S., Anić, I., Hartman, T., Bončina, A. 2008: Longterm changes in tree species composition in old- growth dinaric beech-fir forest. Glasnik za šumarske pokuse, Vol. 42, 13-27, Zagreb.

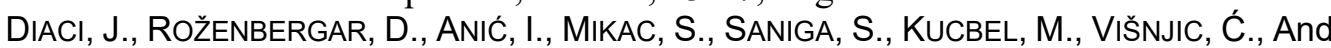
BALLIAN, D. 2011: Structural dynamics and synchronous silver fir decline in mixed old-growth mountain forests in estern and Southeastern Europe. Forestry, 84. 479.491.

Dubravec, T., Čavlović, J., Roth, V., VRbek, B., Novotny, V., Dekanić, S. 2006: The structure and possibility of natural regeneration in managed and non-managed beech and fir forests in Croatia. Periodicum Biologorum, Vol. 108, No 6.

DUBRAVEC, T., DEKANIĆ, S. 2007: Utjecaj strukture sastojine na dinamiku razvoja mladoga naraštaja i potrajnost šume bukve i jele u nacionalnom parku Risnjak. Šumarski list, Zagreb, br. 11-12.

HADŽIABDIĆ, S., VUKOREP, I. 1996: Die Situation der Forstwirtschaft in BosnienHerzegowina. Holz-Zentralblatt 103/104, 1558 .

JÄHNE, S., DOHRENBUSCH, A. 1997: Ein Verfahren zur Beurteilung der Bestandesdiversität. Forstw. Cbl. 116, 222-345.

KORPEL, Š. 1995: Die Urwälder der Westkarpaten. Gustav Fischer Verlag, Stuttgart, Jena, New York.

LEIBUNDGUT, H. 1986: Ziele und Wege der naturnahen Waldwirtschaft. Schweizerische Zeitschrift für Forstwesen (SZF) 137, 245-250.

LeiBundGUt, H. 1993: Europäische Urwälder. Verlag Paul Haupt, Bern, Stuttgart.

MeYer, P., Pogoda, P. 2001: Entwicklung der räumlichen Strukturdiversität in nordwestdeutschen Naturwäldern. AFJZ, 172 (12), 213-220.

MeYeR, P., TABAKU, V., LÜPKE, V. B. 2003: Die Struktur albanischer RotbuchenUrwälder - Ableitungen für eine naturnahe Buchenwirtschaft. Forstw. Cbl. 122, 47-58

MÜLLER, J. 2005: Waldstrukturen als Steuergröße für Artengemeinschaften in kollinen bis submontanen Buchenwäldern. Dissertation, München.

PINTARIĆ, K. 1978: Urwald Perućica als natürliches Forschungslaboratorium. AFJZ 33 (24), 702-707.

PINTARIĆ, K. 1991: Waldbau - Teil II - Verjüngung und Pflege. Sarajevo.

PRPIĆ, B. 1979: Struktur und Funktionen von Buchen-Tannen-Urwäldern (AbietiFagetum illyricum / Horv. 1938/) in der SR Kroatien. In: Tagungsband „II. Kongress jugoslawischer Ökologen“, Zadar-Plitvice, 899-924.

PrPić, B., Selesković, Z., VUKELIĆ, J. 1995: Der Urwald Čorkova Uvala - ein Modell für den multifunktionalen Buchen-Tannen-Plenterwald. In: Eder, W. (Hrsg.) Ergebnisse d. 7. IUFRO Tannensymposiums, Mainz, 250 - 253. 
Sebastia, M.-T., Casalas, P., Vojniković, S., Bogunić, F., Beus, V. 2005: Plant diversity and soil properties in pristine and managed stands from Bosnian mixed forests. Forestry 78 (3), 297-303.

ShanNon, C. E. AND WeAVER, W. 1976: Mathematische Grundlagen der Informationstheorie. München, Wien, Oldenburg

STOJANOVIĆ, O. AND DRINIĆ, P. 1974: Untersuchung zur Größe konzentrischer Probeflächen für die Waldbewertung. Arbeiten der Fakultät für Forstwissenschaften und des Instituts für Forstwirtschaft in Sarajevo 7 (4-6).

TABAKU, V. 1999: Struktur von Buchen-Urwäldern in Albanien im Vergleich mit deutschen Buchen-Naturwaldreservaten und Wirtschaftswäldern. Cuvillier, Göttingen

TeR BRAak, C.F.K., Smilauer, P. 1999-2002: Canoco for Windows 4.5; Biometrics Plant Research International, Wageningen, The Netherlands.

TICHY, L.J. 2007: Juice 6.5 software. Inst. of Botany and Zoology; Masary Univ. Brno, Czech Republic.

VIŠNJIĆ, Ć, VoJNIKOVIĆ, S., IORAS, F., DaUtBašIĆ, M., ABRUdAN, I. V., GUREAN, D., Lojo, A., TREŠtić, T., BAlLIAN, D., BAJRIĆ, M. 2009: Pristine Status Assessment of Plješevica Forest in Bosnia- Herzegovina. Not. Bot. Agrobot. Cluj. 37(2), 22-27.

VIŠnjić, Ć., Solaković, S., Mekić, F., Balić, B., Vojniković, S., DaUtBašić, M., Gurda, S., IORAS, F., Ratnasingam, J., ABrudan, I. V. 2013: Comparison of structure, regeneration and dead wood in pristine forest remnant and managed forest on Grmeč Mountain in Western Bosnia. Plant biosystems 2013 v.147 no.4 pp. 913-922

VIŠnjić, Ć., Balić, B., VoJniković, S., Mekić, F., UzUnović, A. (2015): Structural characteristics, dynamics and texture development of pristine forest Ravna vala on Bjelašnica; Radovi Šumarskog fakulteta Univerziteta u Sarajevu; Šumarski fakultet Univerziteta u Sarajevu Vol. 45. Br. 2, str. 100-112.

VoJNIKOVIĆ, S. 2017: Zaštićena šumska područja u Bosni i Hercegovini / Protected forest areas in Bosnia and Herzegovina; Šumarski fakultet Univerziteta $\mathrm{u}$ Sarajevu, Sarajevo (str.: 1-220).

VRŠKa, T., Hort, L., Odehnalova, P., Adam, D., Horal, D. 2001: The Milešice pristine forest after 24 years $(1972-1996)$. J. For. Sci. 47(6), 255 - 276. 


\section{SAŽETAK}

U ovom radu su predstavljeni rezultati istraživanja uporedne analize strukturnih i vegetacijskih karakteristika kao i stanja mrtvog drveta $u$ prašumskom rezervatu „Ravna vala“ i gospodarskoj šumi bukve i jele (sa smrčom) na planini Bjelašnici. Prašuma "Ravna vala" se nalazi u centralnom dijelu Bosne i Hercegovine, oko 20 km jugozapadno od Sarajeva na planini Bjelašnici, na nadmorskoj visini od 1280 do 1450 m. Gospodarska šuma bukve i jele sa smrčom koja je služila za poređenje se graniči sa prašumom „Ravna vala“ i ima iste stanišne uvjete; klima, orografja i zemljište, i slične strukturne karakteristike i sastav vrsta drveća kao prašuma.

$\mathrm{U}$ istraživanom području dominiraju zajednice bukve i jele (Abieti-Fagetum illyricum TREG. 1957), u kojima se može javiti i smrča. Pojedinačno ili u manjim grupama u uvalama na dubljim zemljištima javljaju se plemeniti lišćari; gorski javor i gorski brijest.

$\mathrm{Na}$ osnovu provedenih istraživanja vidljivo je da prašuma i gospodarska šuma pokazuju prebornu debljinsku strukturu. Međutim, velike razlike između prašume i gospodarske šume, $\mathrm{u}$ distribuciji stabala po debljinskim klasama, se javljaju u završnom debljinskom stepenu. U prašumi je evidentrano stablo sa prsnim prečnikom od $145 \mathrm{~cm}$ dok je prsni prečnik najdebljeg stabla u gospodarskoj šumi $70 \mathrm{~cm}$. Ovo je i očekivano imajući u vidu da je u gospodarskoj šumi u kojoj se gazduje skupinasto prebornim sistemom, završni debljinski stepen za glavne vrste drveća $70-75 \mathrm{~cm}$. Zaliha sastojine u prašumi iznosi $770 \mathrm{~m}^{3} /$ ha i dva puta je veća nego u gospodarskoj šumi. Zaliha mrtvog drveta u prašumi iznosi $170 \mathrm{~m}^{3} /$ ha i pet puta je veća nego $u$ gospodarskoj šumi. Dok su u gospodarskoj šumi u zalihi mrtvog drveta najviše zastupljeni panjevi i deblje grane, u prašumi se najčešće nalaze ostaci debala, visoki panjevi kao i ležeća i stojeća suha stabla. Floristička istraživanja su pokazala da se preborno gazdovanje ne odražava negativno na floristički diverzitet, već je prizemna vegetacija bogatija vrstama u gospodarskoj šumi. Tako Shannonov index diversiteta $i$ index izjednačenost (Evenness) vaskularne flore ima veće vrijednosti u gospodarskoj šumi. Za razliku od florističkog diverziteta, Indeks sastojinskog diverziteta po JÄHNE and DOHRENBUSCH (1997) pokazuje veće vrijednosti u prašumi u odnosu na gospodarsku šumu. Kako je za potrebe obračuna sastojinskog diverziteta uzeto više strukturnih parametara sastojine (diverzitet vrsta, prostorni raspored stabala na površini, vertikalna sastojinska struktura i diferencijacija krošnji stabala), dobijeni rezultati sastojinskog diverziteta u ovome radu ukazuju na potrebu uzimanja više strukturnih i vegetacijskih parametara sastojine za obračun vrsnog i sastojinskog diverziteta i njihove pravilne interpretacije.

Corresponding author: Ćemal Višnjić; Faculty of Forestry University of Sarajevo, Zagrebačka 20, 71000 Sarajevo, Bosnia \& Herzegovina; e-mail: c.visnjic@sfsa.unsa.ba 